\title{
The Human Immunodeficiency Virus sero-Prevalence and AIDS- Related Opportunistic Infection Patterns among Women with Retained Placenta in Eastern Tanzania
}

\author{
Article by Admirabilis Kalolella \\ Ifakara health institute, Dar es salaam, Tanzania \\ E-mail: kalolella@hotmail.com
}

\begin{abstract}
The objective of this study was to characterize women with Retained Placenta (RP) attending obstetric care in eastern Tanzania. We assessed the HIV sero-prevalence, then screened for medical conditions related to RP and described their AIDS related opportunistic infection patterns for the women that were HIV-sero-positive.

The cross sectional study design using medical questionnaires and self-report checklist was used to collect data. The study assessed if there is any relationship between retained placenta and HIV serostatus, and if sero -status and HIV opportunistic infection patterns has relationship with pregnancy outcome and other medical conditions related to pregnancy.

In our study, HIV sero-positive was detected in 105 out of 251 women (42\%). HIV sero-positive women developed prolonged labor, 23(79.3\%), significantly more often compared to HIV sero-negative women, 6 (20.6\%), P value <0.0001. The HIV positive women are significantly more likely to develop Postpartum hemorrhage $(P$ value $=0.0031)$ and to undergo Caesarian section compared to pregnant women without HIV $(P$ value $=0.0113)$. The pregnant women under antenatal ARV Triple $(B+)$ therapy are significantly more likely to end up with preterm delivery $(P$ value $=0.0443)$. Women with HIV progressive symptoms of memory loss, depression, and other neurologic disorders had more underweight babies born $(P-$ Value $=0.0316)$, while women with pneumonia developed vaginal tears more often during delivery $(P-$ Value $=0.0693)$.

In conclusion, RP is common among women with HIV. The prolonged labor is the major medical condition currently faced by HIV infected pregnant women during delivery. Women with HIV progressive symptoms are at risk to give birth to underweight baby and get vaginal tear during delivery. The current $B+A R V$ treatment during antenatal period has positively changed some the pregnancy outcomes among HIV infected pregnant women.
\end{abstract}

Keywords: Retained placenta, sero-positive, HIV sero-status, prolonged labor, HIV opportunistic infection patterns, pregnant women, Post-partum hemorrhage, B+ARV triple therapy.

\section{Introduction}

Maternal HIV infection increases the risk of adverse pregnancy outcome including stillbirth and premature delivery, risk factors for retained placenta (RP) that leads to Post-Partum Haemorrhage (PPH), the major killer of women (Calvert \& Ronsmans, 2015; Kumar, 2016; Slyker et al., 2014). About 13 million premature deliveries occur each year worldwide that constitute of live births $11 \%$, most of them occur in developing countries (Slyker et al., 2014). Sub-Saharan Africa contributes more than one-fourth of these death making the region with the highest incidence of stillbirth in the world (Kumar, 2016; Slyker et al., 2014). The Sub Saharan Africa, experience the highest levels of HIV/AIDS, while millions of women of reproductive age currently live with HIV in sub-Saharan Africa. In Tanzania, more that 7\% of pregnant women are infected with HIV that probably affects the current situations of retained placenta in Tanzania (PMTCT Tanzania, 2016). To reduce adverse effects of HIV/AIDS during pregnancy, the country is implementing new B+ ARV triple therapy as a new program for antenatal services for pregnant 
DOI: $10.21522 / \mathrm{TIJNR} .2015 .03 .02 . A r t 022$

ISSN: $2520-3126$

women infected with HIV, regardless of their CD4 count (PMTCT Tanzania, 2016; Ngarina et al., 2014). In this regime, pregnant women who are sero-positive, with gestation age of 34 weeks and above are given three ARV medicine; Zidovudine + Lamivudine + Nevirapine /Nelfinavir (PMTCT Tanzania, 2016; Ngarina et al., 2014). This regime has been practiced for the past three years in the country.

Retained placenta in humans is referred as a placenta that has not undergone completely expulsion within 60 minutes after the baby's birth when the third stage of labor, between child delivery to the complete expulsion of placenta has been managed actively (Marshall \& Raynor (2014; Agrawal et al., 2013). When placenta is partially separates, the uterus cannot contract properly to compress blood vessels, and therefore the blood vessels inside the uterus will continue to bleed without stopping that result into PPH (Marshall\& Raynor, 2014; Kumar, 2016). Retained placenta brings risks of excessive hemorrhage and infection leading to puerperal sepsis (Kumar 2016; Marshall\& Raynor, 2014; Agrawal et al., 2013).

Studies indicate that pregnant women infected with HIV are at risk to give birth with gestation age of less than 28 weeks, which is called premature delivery (Slyker et al., 2014). Baby born with gestation age around or less than 28 weeks are always born as dead in the form of fresh stillbirth or macerated stillbirth which are risk factors for RP (Marshall\& Raynor, 2014; Kumar, 2016). Women with HIV infection are also at risk to deliver premature baby, one of the major factors contributing to RP (Slyker et al., 2014). The HIV and AIDS is the major cause of death that effect women of reproductive age in sub Saharan Africa, and the region experience the highest levels of HIV/AIDS infection and also highest maternal mortality much of the incidence presumably occur due to HIV infection (Calvert \& Ronsmans, 2015). Considering that millions of women of reproductive age currently living with HIV in sub-Saharan Africa, HIV and AIDS- related conditions may be responsible for a large number of stillbirths (Calvert \& Ronsmans, 2015). Some studies argued that pregnancy fuels advancement of HIV infection, while other observed increased obstetric complications among women living with HIV (Calvert \& Ronsmans, 2015).

Retained placenta is the main causes of PPH in developing countries where access to appropriate obstetrical care is limited (Slyker et al., 2014; Kumar, 2016). Studies indicate that independent risk factors associated with retained placenta include; inadequate utilization of antenatal health care services, history of previous uterine scar due to retained placenta, induced labor, caesarean section, delivery with full urine bladder, uterine abnormality, previous dilatation and curettage, pregnancy at age more than 35 years, many births during lifetime, preterm delivery and placenta with less than $501 \mathrm{~g}$ in weight (Marshall \& Raynor, 2014). Retained placenta membranes and tissue are responsible for postpartum hemorrhage (Kumar, 2016). The treatment of placenta is manual remove of placenta under general anesthesia. The removal of placenta is performed in operating theater with specialized medical staffs that are seldom available in rural areas (Duffy at el., 2014; Kumar 2016; Marshall\& Raynor, 2014).

PPH is defined as the excessive loss of blood after vaginal delivery of new born that is observed from the time of delivery up to forty-two days postpartum (Kumar, 2016; Marshall \& Raynor, 2014). The primary PPH is the most common which is defined as the blood loss estimated more that 500ml within the first twenty-four hours after delivery, while secondary PPH is the bleeding after twenty four hours up to six weeks after delivery (Marshall\& Raynor, 2014). The PPH may pose serious threat of death particularly to women with weak health status regardless of amount of blood loss (Marshall\& Raynor, 2014; Kumar, 2016). The PPH may be caused by failure of the uterus to contract adequately after delivery of baby (uterus atony), retained placenta, traumatized delivery canal, rupture of uterus, or maternal bleeding disorders (Kumar, 2016; Marshall \& Raynor, 2014). The most common treatment for PPH is to ensure stability of hemodynamic through intravenous fluid infusion and blood transfusion to replace lost blood and removing causative factors that exacerbate the situation (Kumar, 2016; Marshall\& Raynor, 2014). Such services are not always available in health facilities of rural area in resource poor countries where many women attend for delivery services and thus the need for pre-diagnostic efforts to prevent unexpected obstetric complication (Slyker et al., 2014; Illah et al., 2013).

It is estimated that $4 \%$ of maternal mortality in Sub-Saharan Africa are related to HIV infected persons 
(Calvert \& Ronsmans, 2015). According to Calvert \& Ronsmans (2015), HIV positive pregnant women are 8 times more likely to die compared to uninfected women.

The investigation that leads to identifying predictors of RP will serve women from unexpected adverse health conditions. Women that are pre-diagnosed of RP at antenatal had a lower blood loss in the range of $100-15000 \mathrm{ml}$ and required less volume of transfusion compared to other women with blood loss around 2500-17000ml (Tikkanen et al., 2011). The relationship of HIV status and RP is still under debate. Limited knowledge is available on relationship of HIV/AIDS to RP. The HIV /AIDS may be responsible for a large number of women with retained placenta. This investigation will help to find out if HIV/AIDS is one among predictors of RP.

Rationale. The RP is one of the leading causes of maternal hemorrhage contributing to high maternal mortality rate worldwide (Illah et al., 2013; Marshall \& Raynor, 2014). The limited health care resources and inadequacy of obstetric care, particularly in the rural areas fuels adverse effect of RP in Tanzania (Illah et al., 2013). In Tanzania, the prevalence of HIV among women who attend ANC is high that could probably affect the current situations of RP (PMTCT Tanzania, 2016). While many studies have previously assessed the relationship of clinical factors to retained placenta, and others assessed the relationship of stillbirth and premature delivery to HIV status, and several other studies are known to be in progress, the vast majority of these studies have been conducted exclusively in still birth, premature delivery in relation to HIV status without looking at HIV progress factors and patterns of AIDS related conditions and opportunistic infection (Slyker et al., 2014; Calvert \& Ronsmans, 2015). With the current changing context in health care technology, provision of health care services and life styles, risk factors for RP will be re-visited to see changes and magnitudes among these factors. Given the high proportion of HIV/AIDS among pregnant women in Tanzania, identifying predictors of RP is critical to reduce consequences of this obstetric complication.

This study examined the relationship between RP and HIV, the magnitude of clinical conditions - HIV progressive factor leading to RP in a Tanzanian context. The study established new way to pre-diagnose or predict RP through combination of medical conditions including serological, AIDS related conditions and its progressive factors. Identifying predictors of RP through medical symptoms will help health care workers to predict, forecast and plan for safe deliveries to serve women from unexpected health problems, reduce $\mathrm{PPH}$, blood transfusion cases and maternal mortality in the country.

The goal of this study was to characterize women with RP that presented to the hospital for obstetric care in eastern Tanzania. This was achieved as follows: We assessed the HIV sero-prevalence among women with RP, we described their AIDS related opportunistic infection progressive patterns of women with RP that are HIV-sero-positive, and we screened for other medical conditions related to RP.

Hypothesis: If HIV infected pregnant women are likely to deliver still birth and pre- term baby, risk factors for RP, then we hypothesize that with the current high prevalence of HIV infection in Tanzania, the HIV status and AIDS- related opportunistic infections are likely to emerge as main predictors of RP. For the past three years Tanzania has been implementing B+ Triple therapy for all pregnant women we also expected changes in pregnancy outcomes. All HIV infected women in Tanzania are required to start B+ ARV triple therapy, thus all women in this study were covered by B+ Triple ARV therapy as part of PMTCT program in Tanzania. We expected to find a high HIV sero-prevalence among women with RP, but also anticipated to find positive effect of ARV therapy counteracting HIV effect on clinical symptoms and pregnancy outcome.

\section{Methodology}

The cross sectional study design was used to collected descriptive data of 251 post- partum women with RP from maternity or labor wards in hospitals providing obstetric care in municipalities of Ubungo, Ilala, Temeke, and Kinondoni, that are located in eastern Tanzania. The data collection was conducted from September 2016 up to February 2017.The study investigated HIV sero-prevalence, HIV/AIDSopportunistic infection patterns and other clinical conditions related to RP. These municipalities have high 
DOI: $10.21522 / \mathrm{TIJNR} .2015 .03 .02 . A r t 022$

ISSN: $2520-3126$

HIV sero-prevalence and high number of deliveries. Women diagnosed or treated for RP formed the population of this study. Eligible participants were obtained from labor or maternity wards in hospitals. The patient was approached by a recruiter to determine her eligibility and recruited by registering her name and giving study identity number. All interested patients were directed to interviewers for the consent process. Informed consent was administered in local language and these consented were asked to undergo rapid clinical and laboratory assessment and interviews. Every patient diagnosed with RP was recruited sequentially to achieve an enrollment of sample size required. This design provided an estimated proportion of women with RP seeking obstetric care that actually have HIV infection, medical conditions related to RP, and description of AIDS- related conditions and opportunistic infection progress and patterns in patient with HIV infection. The HIV progress factors were divided into two categories: 1. HIV early stage symptoms (WHO clinical stages 1\&2) and, 2. HIV late (AIDS) stage symptoms (WHO clinical stages $3 \& 4)$. The study also collected information on general description of medical conditions among women with and without HIV infection.

The consented post- partum women with RP undergone clinical assessment to identify clinical conditions, signs and symptoms of opportunistic or HIV/AIDS related infections and other medical conditions. Rapid HIV test was done using HIV SD Bioline (SD South Korea) to determine sero-status. The HIV sero-status was confirmed by Uni-Gold HIV Rapid Test Kits, Trinity Biotech. The Participants were then clinical assessed and interviewed for self-report of clinical signs to determine HIV/AIDS progressive symptom patterns and severity.

Clients were enrolled for the study only if;

- they were diagnosed with retained placenta by hospital staffs or completed treatment for RP

- they consents to participate

- Potential participants were excluded if they were

- presenting with danger sign or signs of severe illness such as inability to speak, drink, and vomiting

- recent history of convulsions or eclampsia

- lethargic or unconscious

- Experiencing danger signs, or other conditions requiring immediately treatment.

The Medical questionnaire for Medical conditions, signs and symptoms of AIDS related complication was used to collect data. The first part of the questionnaire collected information on the demographic characteristic; age, marital status, education level, and number of births. The second and third part was checklist for sign and symptoms for HIV/AIDS related infections, while the fourth part was medical conditions checklist. Each question of checklist was defined as "Yes" if sign and symptom present or "No" if there was no such symptom. The last section collected information on HIV status and pregnancy outcomes.

\section{Ethical consideration}

The Tanzanian Medical Research Coordinating Committee of National Institute for Medical Research (NIMR) approved the study. The informed consent was administered to every postpartum woman with RP interested to participate in the study.

\section{Data analysis}

The data entry screen was developed from EpiData software. The data that were entered into the software were transferred to SAS version 9.4 for data analysis. The primary variables of interest were; HIV sero-prevalence among women with retained placenta. HIV/AIDS related symptoms and its progressive factors among women with reined placenta who are HIV positive. Medical conditions among women with RP were also documented to all patients regardless of their HIV status and then compared. Variables were categorized as: age, marital status, education level, and number of birth of women with 
retained placenta, HIV status, HIV early stage symptoms (WHO clinical stages $1 \& 2$ ), HIV late (AIDS) stage symptoms (WHO clinical stages 3\&4), other medical conditions that may be associated with RP, and pregnancy outcomes. Frequencies tables and cross tabulations were used to determine associations between variables. The chi- squire test was used to analyze categorical data to test statistical significance between these differences or association between variables. The association or the differences were considered significant if $\mathrm{P}$-values $<0.05$.

The early HIV stage symptoms observed were; fever, chill, night sweats, rashes, productive cough, muscle aches, sore throat, fatigue, swollen lymph nodes and mouth ulcers (WHO, 2015). The late stage symptoms are; rapid weight loss, recurring fever or profuse night sweats, extreme and unexplained tiredness, prolonged swollen lymph glands in the armpits, groin or neck, diarrhea that lasts for more than a week, sores of the mouth, anus, or genitals, pneumonia, red, brown, pink or purple blotches on or under the skin or inside the mouth, nose or eyelids, memory loss and depression and neurological disorders and Kaposi's sarcoma or lymphoma (WHO, 2015).

\section{Results}

A total of 251 women with retained placenta were screened for HIV status, HIV/ AIDS related symptoms, other medical conditions and pregnancy outcomes, and the information were included for data analysis. Among women participated, 46 (18\%) were aged between 18-25 years, and 196 (78\%) aged between 26- 40, while $9(3.6 \%)$ of them were women aged 41-44 (Table 1). The large proportion of them, $185(74 \%)$ had 1-3 births; while 62(24\%) women had 4-6 births, and 4 of them (1.5\%) had delivered 7-9 times. Majority of them, 169(67\%) achieved primary level education, while 59(23.5\%) completed secondary education, $20(8 \%)$ of these women had not attended any school, and only $3(1.2 \%)$ completed college education (Table 1).

The results indicate that among 251 pregnant women with retained placenta 105(41.8\%) were seropositive, while $146(58.1 \%)$ are sero-negative (Table 2). Pregnant women aged 26-40years, that are 79 (31.4\%) are more affected with HIV followed by the age of 18-25 years, 22(8.7\%), however, there is no significance difference of HIV prevalence among different groups of pregnant women (Table 2). In this regard, among pregnant women, the prevalence of HIV is independent of age, which is different among the women in general public where HIV prevalence is dependent of age groups.

Women with retained placenta that are HIV sero-positive reported significant more episodes of caesarean section operation, 21(61.7\%) during delivery compared to women that are sero-negative, 13(38.2\%) (P value=0.0113) (Table 3). Women with HIV have significantly more developed PPH 19(67.8\%) compared to women without HIV, 9(32.1\%) (P value=0.0031). (Table 3). The pregnant women with retained placenta that are sero-positive show significant more preterm deliveries $10(66.6 \%)$ compared to women who are sero-negative, 5(33.3\%), $\mathrm{P}$ value $=0.0443$. Women with HIV had less occasions of still born babies 4(28.5\%), than women without HIV, 10(71.4\%), however the association was not significant.

There are no significant differences in their baby weights and stillborn births among them probably due to ARV triple therapy current being received by all HIV- positive pregnant women in Tanzania (Table 3 ). Triple ARV therapy may probably enhanced baby weight during pregnancy. To the surprise, women without HIV show slightly higher number of still born baby deliveries compared to Women with HIV although the difference is not significant. In this situation, ARV triple therapy may have reduced still born babies among HIV sero-positive women. The occurrence of prolonged labor was more significant among HIV infected women 23(79.3\%), compared to HIV negative women 6(20.6\%), P-value <0.0001(Table $3)$. The study indicates new findings in pregnancy outcomes opposing other study findings where HIV sero-positive pregnant women were observed to deliver low weight and stillborn babies significantly more often compared to HIV sero-negative (WHO, 2015; Calvert \& Ronsmans, 2015). The previous episodes of retained placenta and history of myomectomy had no significant association with retained placenta in women of both group of HIV negative and positive (Table 3). 
DOI: $10.21522 / \mathrm{TIJNR} .2015 .03 .02 . A r t 022$

ISSN: $2520-3126$

The findings show that women with common HIV progressive persistent pneumonia which is among the late HIV/ AIDS stage (WHO clinical stage 3 \&4) symptoms had vaginal tears during birth more often (35.2\%) than did women who had no pneumonia during delivery (18.9\%), P- Value $=0.0693$ (Table 4). Women with memory loss, depression, and other neurologic disorders had more babies born with body weight below $2500 \mathrm{~g}$ (underweight baby) (20.8\%) compared to women without these symptoms $(6.1 \%)$, P- Value 0.0316 (Table 4). The symptoms of memory loss, depression, and other neurologic disorders are among the HIV progressive symptoms for individual with HIV in late AIDS stage (WHO clinical stage 3 \&4). Other HI/AIDS related progressive symptoms had no significant association with pregnancy outcomes.

\section{Discussion}

This study was conducted in public health facilities providing obstetric care in eastern Tanzania where low income women attend. The information was collected in municipalities with higher rate of HIV transmission. In Tanzania, almost $7 \%$ of pregnant women are infected with HIV, and all of them are expected to be on Prophylaxis ARV (B+) Triple therapy from antenatal clinics (PMTCT Tanzania, 2016, Ngarina et al., 2014). To understand the prevalence of HIV among women with the retained placenta is important step to inform health care workers to predict the possibility RP for treatment and prevention of PPH to reduce mortality.

Among 251 women with retained placenta, 105 (42\%) were HIV sero-positive. This indicates that RP is common among HIV infected pregnant women. The study indicates that prevalence of HIV among pregnant women with retained placenta is independent of age group; this is opposite when HIV prevalence is assessed through age groups in adult women without pregnancy (WHO, 2015; Calvert \& Ronsmans, 2015). Women with retained placenta that are HIV sero-positive are more likely to develop $\mathrm{PPH}$ as well as to undergo caesarean section operation compared to women that are sero-negative. Following ARV triple therapy, women with HIV had less occasions of still born babies compared to women without HIV although the association was not significant. The pregnant women with retained placenta that are sero-positive under ARV therapy show significant more preterm deliveries compared to women who are sero-negative. This finding corresponds to other similar studies (Calvert \& Ronsmans, 2015; Slyker et al., 2014). This finding of increased preterm deliveries were observed in various studies indicating that ARV therapy excessively increased preterm deliveries among HIV infected mothers (Aniji et al., 2013; Watts et al., 2013; Short et al., 2014). Women without HIV show slightly higher percentage of still born baby deliveries compared to Women with HIV although the difference is not significant. We observed no significant differences in their baby weights and stillborn births among women with different HIV status. The ARV triples therapy current being administered to all pregnant women that are seropositive in antenatal clinics may have influenced some of the observed results.

In the medical conditions, we observed phenomenon of prolonged labor (prolonged second or third stage of labor) that was more significant among HIV infected women. Study shows that women with HIV are significantly more likely to experience PPH and to undergo Caesarian section compared to women without HIV.

The pattern of AIDS related opportunistic infections among women with RP that are HIV-sero-positive indicate various pregnancy outcomes. Women with HIV progressive symptom of pneumonia which is among the late HIV/ AIDS stage (WHO clinical stage $3 \& 4$ ) symptoms are more likely to develop vaginal tears during birth than women without pneumonia. Women with memory loss, depression, and other neurologic disorders are more likely to give birth to underweight babies compared to women without these symptoms. The symptoms of memory loss, depression, and other neurologic disorders develop among HIV infected person during late AIDS stage (WHO clinical stage $3 \& 4$ ). Other HIV/AIDS related progressive symptoms had no significant association with pregnancy outcomes. 


\section{Conclusion}

The RP is common among women with HIV. The prolonged labor is the major medical condition currently faced by sero-positive pregnant women during delivery that need attention from health care workers. Women with retained placenta that are HIV sero-positive is more likely to develop PPH as well as to undergo caesarean section operation compared to women that are sero-negative. Majority of HIV Infected women develop prolonged labor when giving birth. The HIV/AIDS progressive symptom of pneumonia put a woman at risk to develop vaginal tear during delivery, while symptoms of memory loss, depression, and other neurologic disorders subject pregnant women at risk of giving birth to underweight baby. The current $\mathrm{B}+$ treatment during antenatal period has changed positively some of the pregnancy outcomes among HIV positive pregnant women.

\section{Acknowledgements}

We would like to thank and express our sincere gratitude to the municipal medical officers, in-charges of municipal hospital and maternity wards of these hospitals, medical doctors and midwifes of all maternity sections and all participants for their support and their willingness participate and disclose their clinical symptoms and to accept this study.

Table 1. The demographic characteristics of women with retained placenta $(\mathrm{n}=251)$

\begin{tabular}{|l|l|l|l|}
\hline Demographic backgrounds & \multicolumn{4}{l|}{} \\
\hline Variable & Response & Frequency & Percent \\
\hline Age group in years & $18-25$ & 46 & $18 \%$ \\
\hline & $26-40$ & 196 & $78 \%$ \\
\hline Number of births & $41-44$ & 9 & $3.6 \%$ \\
\hline & $1-3$ & 185 & $74 \%$ \\
\hline & $4-6$ & 62 & $24 \%$ \\
\hline Education level & $7-9$ & 4 & $1.5 \%$ \\
\hline & Primary & 169 & $67 \%$ \\
\hline & Secondary & 59 & $23.5 \%$ \\
\hline & College & 3 & $1.2 \%$ \\
\hline Total & None & 20 & $8 \%$ \\
\hline
\end{tabular}

Table 2. The HIV status among women with Retained placenta based on the age group $(\mathrm{n}=251)$

\begin{tabular}{|l|l|l|l|}
\hline \multirow{2}{*}{ Age Group } & \multicolumn{2}{|l|}{ HIV status } & \multirow{2}{*}{ P value } \\
\cline { 2 - 3 } & HIV Negative $(\mathrm{n}=146)$ & HIV Positive $(\mathrm{n}=105)$ & \\
\cline { 2 - 3 } & $(\mathrm{n}, \%)$ & $(\mathrm{n}, \%)$ & \\
\hline $18-25$ & $24(9.56)$ & $22(8.76)$ & \\
\hline $26-40$ & $117(46.61)$ & $79(31.47)$ & \\
\hline $41-44$ & $5(1.99)$ & $4(1.59)$ & \\
\hline Total & $146(58.1)$ & $105(41.8)$ & \\
\hline
\end{tabular}


DOI: $10.21522 /$ TIJNR.2015.03.02.Art022

ISSN: $2520-3126$

Table 3. The Common medical conditions among women with retained placenta based on HIV status $(n=251)$

\begin{tabular}{|c|c|c|c|}
\hline \multirow{3}{*}{$\begin{array}{l}\text { Medical } \\
\text { conditions }\end{array}$} & \multicolumn{2}{|c|}{ HIV status $n=251$} & \multirow[t]{3}{*}{$\mathrm{P}$ value } \\
\hline & HIV Negative & HIV positive & \\
\hline & $\mathrm{n},(\%)$ & $\mathrm{n},(\%)$ & \\
\hline $\begin{array}{l}\text { Caesarean } \\
\text { section }\end{array}$ & $13(38.2)$ & $21(61.7)$ & 0.0113 \\
\hline Myomectomy & $2(40)$ & $3(60)$ & 0.4055 \\
\hline $\mathrm{PPH}$ & $9(32.1)$ & $19(67.8)$ & 0.0031 \\
\hline Baby weight & $11(52.3)$ & $10(47.6)$ & 0.5744 \\
\hline $\begin{array}{l}\text { Prematurely } \\
\text { delivery }\end{array}$ & $5(33.3)$ & $10(66.6)$ & 0.0443 \\
\hline Prolonged labor & $6(20.6)$ & $23(79.3)$ & $<0.0001$ \\
\hline Stillborn baby & $10(71.4)$ & $4(28.5)$ & 0.3006 \\
\hline $\begin{array}{l}\text { Previous } \\
\text { retained placenta }\end{array}$ & $7(43.7)$ & $9(56.2)$ & 0.227 \\
\hline
\end{tabular}


泀

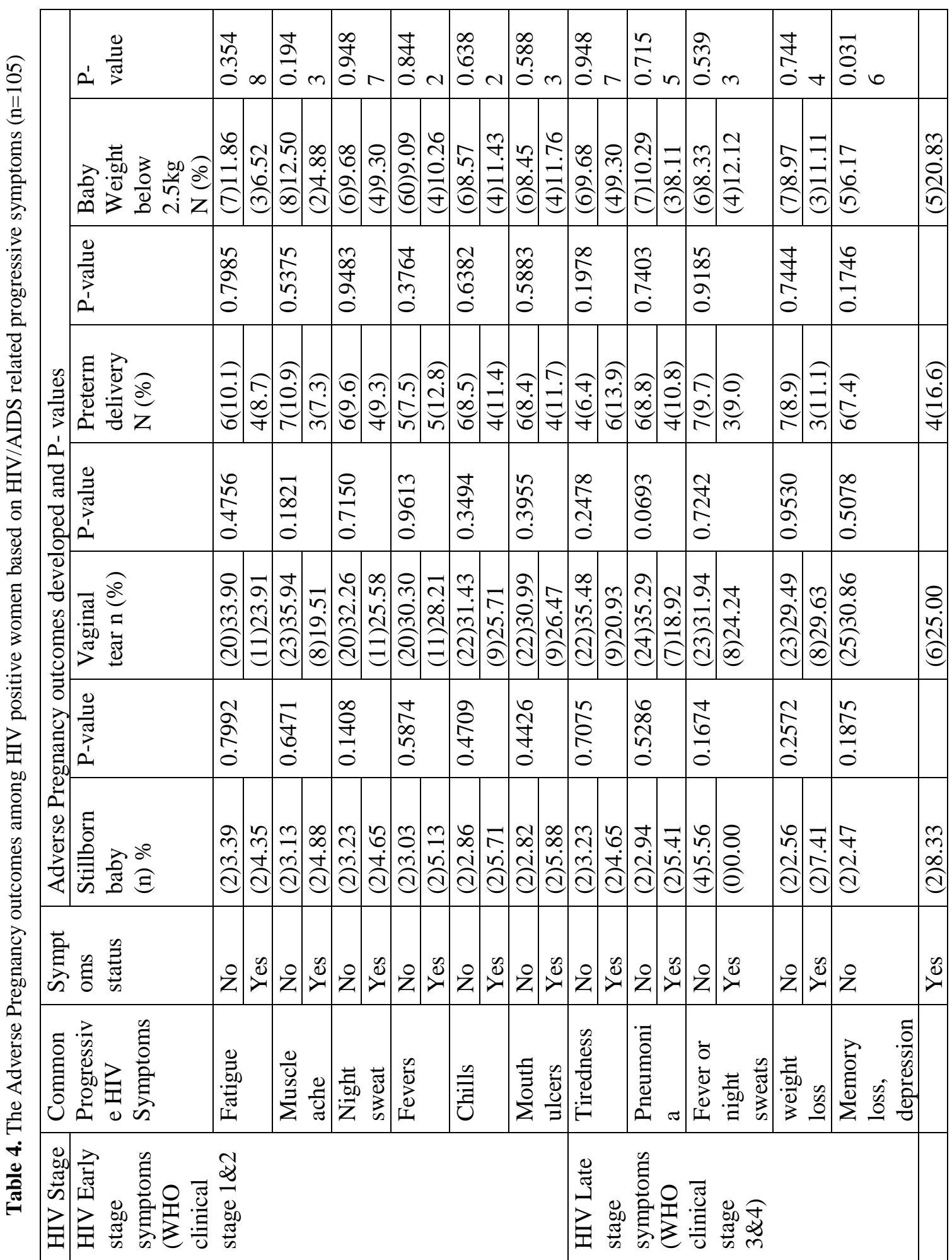


DOI: $10.21522 /$ TIJNR.2015.03.02.Art022

ISSN: $2520-3126$

\section{References}

[1]. Agrawal, A. Thakur, A. Rijal, P. Basnet, P. Ghimire, A \& Uprety, D. (2013). Management of retained placenta in patient with valvular heart disease with pulmonary oedema. Health Renaissance, 201(1), 199-205.

[2]. Aniji C D,1 FCOG (SA); O A Towobola,1 PhD; M E Hoque,2 MSc; T J Mashamba,1 MB ChB; S Monokoane,1 FCOG (SA) (2013). Impact of antiretroviral therapy on pregnancy outcomes. S Afr J HIV Med 2013; 14(4):176-178. DOI:10.7196/SAJHIVMED.834.

[3]. Calvert C, Ronsmans C. (2015). Pregnancy and HIV disease progression: a systematic review and metaanalysis. Journal of Tropical Medicine and International Health, 20(2):122-45. Retrieved June 5, 2016, from PubMed.

[4]. Illah E, Mbaruku G, Masanja H, Kahn K. (2013). Causes and risk factors for maternal mortality in rural Tanzania--case of Rufiji Health and Demographic Surveillance Site (HDSS). Afr J Reprod Health. 2013 Sep; 17(3):119-30.

[5]. Jayne E, Marshall, Maureen D Raynor (2014). Myles' Textbook for Midwives, $16^{\text {th }}$ Edition, UK: Elsevier Health Sciences.

[6]. Jennifer A Slyker, Janna Patterson, Gwen Ambler, Barbra A Richardson, Elizabeth Maleche-Obimbo, Rose Bosire, Dorothy Mbori-Ngacha, Carey Farquhar, and Grace John-Stewart (2014). Correlates and outcomes of preterm birth, low birth weight, and small for gestational age in HIV-exposed uninfected infants. BMC Pregnancy and Childbirth 201414:7. DOI: 10.1186/1471-2393-14-7.

[7]. Kumar, N. (2016). Postpartum Hemorrhage; a Major Killer of Woman: Review of Current Scenario. Obstetrics \& Gynecology International Journal, 4(4). Published online.

[8]. Ngarina M, Tarimo EAM, Naburi H, Kilewo C, Mwanyika-Sando M, Chalamilla G, et al. (2014) Women's Preferences Regarding Infant or Maternal Antiretroviral Prophylaxis for Prevention of Mother-To-Child Transmission of HIV during Breastfeeding and Their Views on Option B+ in Dar es Salaam, Tanzania. PLoS ONE 9(1): e85310. doi:10.1371/journal.pone.0085310.

[9]. PMTCT Tanzania. National resource centre for prevention of mother to child HIV transmission (2016). Retrieved June 152016 from http://pmtct.or.tz/pmtct-tanzania/pmtct-in-tanzania/.

[10]. Short C-ES, M Douglas, JH Smith and GP Taylor (2014) Preterm delivery risk in women initiating antiretroviral therapy to prevent HIV mother-to-child transmission. HIV medicine 201415, 233-238. DOI: 10.111/hiv.12083.

[11]. Tikkanen $\mathrm{M}^{1}$, Paavonen J, Loukovaara M, \& Stefanovic V. (2011) Antenatal diagnosis of placenta accreta leads to reduced blood loss. Journal of Acta Obstetricia et Gynecologica Scandinavica, 90(10), 1140-6.

[12]. Watts DH ${ }^{1}$, Williams PL, Kacanek D, Griner R, Rich K, Hazra R, Mofenson LM, Mendez HA; Pediatric HIV/AIDS Cohort Study (2013). Combination antiretroviral use and preterm birth. J Infect Dis. 2013 Feb 15; 207(4):612-21. doi: 10.1093/infdis/jis728.

[13]. WHO (2015) Guideline on when to start antiretroviral therapy and on pre Exposure prophylaxis for HIV (pdf). Retrieved June 152016 from http://www.who.int/hiv/pub/guidelines/clinicalstaging.pdf). 